\title{
Novel coronavirus associated with severe respiratory disease: Case definition and public health measures
}

N Danielsson (Niklas.Danielsson@ecdc.europa.eu) ${ }^{1}$, on behalf of the ECDC Internal Response Team ${ }^{2}$, M Catchpole ${ }^{3}$

1. European Centre for Disease Prevention and Control, Stockholm, Sweden

2. The members of the team are listed at the end of the article

3. Health Protection Agency, London, United Kingdom

Citation style for this article:

Danielsson N, on behalf of the ECDC Internal Response Team, Catchpole M. Novel coronavirus associated with severe respiratory disease: Case definition and public health measures . Euro Surveill. 2012;17(39):pii=20282. Available online: http://www.eurosurveillance.org/ViewArticle.aspx?Articleld=20282

Article submitted on 27 September 2012 / published on 27 September 2012

Two cases of rapidly progressive acute respiratory infection in adults associated with a novel coronavirus have generated an international public health response. The two infections were acquired three months apart, probably in Saudi Arabia and Qatar. An interim case definition has been elaborated and was published on the World Health Organization website on 25 September 2012.

\section{Case 1}

On 13 June 2012 a patient in their sixties presented with deteriorating pneumonia in Jeddah, Saudi Arabia and a seven day history of respiratory symptoms. The patient developed acute renal failure and died on 24 June 2012. A novel beta-coronavirus was isolated in Saudi Arabia* and sequenced at the Erasmus Medical Centre (EMC) in Rotterdam, the Netherlands [1].

\section{Case 2}

On 11 September 2012 a patient in their forties with severe respiratory symptoms was evacuated from Qatar to a United Kingdom hospital and was admitted to intensive care there on 12 September. The patient remains in hospital and has been on life support with pulmonary and renal failure. Extensive diagnostic tests for a causative agent were negative but on 21 September a pan-coronavirus RT-PCR test performed on lower respiratory samples was positive for a conserved sequence of the coronavirus polymerase gene [2]. Comparison with the nucleotide sequence at the EMC indicated a close match with the novel virus isolated from Case 1. Contacts of Case 2, many of them healthcare workers, are being actively identified, monitored and investigated for coronavirus infection. Some of them have reported mild respiratory symptoms but none have tested positive for the novel virus or developed severe disease to date [3].

\section{Background}

Coronaviruses are globally distributed and are found in humans, other mammals and birds. They are enveloped RNA viruses classified in alpha, beta and gamma genera. Up to one third of mild upper respiratory tract infections in adults are caused by human coronaviruses. The zoonotic severe acute respiratory syndrome (SARS) beta-coronavirus (SARS-CoV) caused the SARS outbreak in 2003 when over 900 people died. [4] Human coronaviruses are transmitted through direct contact with secretions and via aerosol droplets. Infected patients also excrete virus in faeces and urine and under certain circumstances, airborne transmission can occur from aerosolised respiratory secretions and faecal material [5].

The detection of a novel coronavirus associated with severe respiratory disease and renal failure requires urgent assessment and careful management. The United Kingdom Health Protection Agency (HPA) alerted European Union (EU) Member States and other countries via the Early Warning and Response System (EWRS) and International Health Regulations (IHR) mechanisms.

\section{Control measures}

The HPA has recommended stringent control measures and developed an early case definition [6]. The European Centre for Disease Prevention and Control (ECDC) has developed a risk assessment in response to the cases [2]. A surveillance strategy has been agreed between ECDC and WHO with the first priority being to determine whether there are additional severe cases. The initial virology results and the separation in time of the only two confirmed cases suggest an infection that quite probably is of zoonotic origin and different in behaviour from SARS [5]. It is essential to rule out there being additional severe undiagnosed cases, especially since the transfer of severely ill patients in air ambulances meant that cases may be missed by conventional surveillance that is based on clinical notification by the original diagnosing physician, particularly primary care physicians. Hence the interim case definition has been developed with the aim of providing a high level of sensitivity for identifying cases ill 
enough to require hospital care or having pneumonia while avoiding cases with only mild symptoms [7].

\section{Case definition}

The case definition applies the established link that both cases stayed in the Arabian Peninsula but makes it conditional of hospitalisation or pneumonia, which means that cases with a link to an affected area but only mild symptoms do not require investigation. The affected area is currently defined as Saudi Arabia and Qatar but can be expanded as needed. Human coronaviruses have a short incubation period of 3 to 4 days. The longest incubation period observed during the SARS outbreak was 12 days. However, this was an outlier and a pragmatic incubation period of up to 10 days has been adopted for the case definition. The case definition should be used by clinicians for deciding which patients require investigation for possible novel coronavirus infection and which patients should be reported to national authorities. An interim case definition was published on the WHO website on 25 September [8]. It is expected to be amended once more epidemiological and diagnostic information becomes available and clinicians and public health managers should stay updated with the latest version on the website.

EU Member States have been requested to report patients meeting the case definition to ECDC through the EWRS and countries should continue to report probable or confirmed cases through the IHR contacts at WHO regional offices as mandated by the IHR. There is currently no rapid diagnostic test that easily confirms infection with this novel virus. Virus detection and serological testing is being developed by the HPA, the EMC and the University of Bonn, Germany and this was facilitated through close collaboration including the provision of preliminary sequences and a virus isolate between those institutions [9].

\section{Infection control advice}

The HPA has developed specific infection control advice for suspected or confirmed novel coronavirus cases. The guidelines take a strict precautionary approach, whereby patients are isolated in negative-pressure single rooms or, if this is not possible then a single room with en-suite facilities. Full personal protective equipment (PPE), including gowns, gloves and FFP 3 masks are worn by staff and others having direct contact with the patient [6].

\section{Conclusions}

This situation is still evolving and there are many unknowns to consider in hypothesis generation and control measures. There is strong evidence that a novel virus caused the severe disease in the two patients. Based on this assumption it can be concluded that the virus poses an as yet poorly defined level of threat to people's health. There may have been other cases in the past that were missed and serological testing of stored sera and other specimens from such cases will be important. Serological testing will also determine whether the two cases represent the most severe end of a spectrum of clinical presentations which also includes mild and asymptomatic infections or if they are isolated events. To date, the long period between occurrence of the two cases and the lack of secondary cases among contacts suggest the disease is poorly communicable in humans. Our assessment, based on the limited information currently available, is that the risk of wide spread transmission resulting in severe disease is low. However, the emergence of a novel coronavirus requires a thorough assessment which is currently being coordinated at international level.

\section{The ECDC internal response team}

Katrin Leitmeyer, Pete Kinross, Herve Zeller, Niklas Danielsson, Pasi Penttinen, Rene Snacken, Anna-Pelagia Magiorakos, Amanda Ozin, Romit Jain, Eve Robinson, Lara Payne Hellstrom, Angus Nicoll, Josep Jansa and Denis Coulombier.

\section{*Authors' correction:}

The country in which the virus was isolated was added on 28 September 2012 at the request of the authors.

\section{References}

1. ProMED-mail. Novel coronavirus - Saudi Arabia: human isolate Archive Number: 20120920.1302733 20 September 2012 21:51:30 CEST. Available from: http://www.promedmail.org/ direct.php?id=20120920.1302733

2. European Centre for Disease Prvention and Control (ECDC). Rapid Risk Assessment . Severe respiratory disease associated with a novel coronavirus. Stockholm: ECDC; 2012. [Accessed 26 Sep 2012]. Available from: http://ecdc.europa. eu/en/publications/Publications/RRA-Novel-coronavirusfinal20120924.pdf

3. Health Protection Agency (HPA). HPA Press release. Acute respiratory illness associated with a new virus identified in the UK. London:HPA; 2012. [Accessed 25 Sept 2012]. Available from: http://www.hpa.org.uk/NewsCentre/ NationalPressReleases/2012PressReleases/120923acuterespir atoryillnessidentified/

4. World Health Organization (WHO). WHO final summary SARS, 15 August 2003:Summary table of SARS cases by country, 1 November 2002 - 7 August 2003. Geneva: WHO; 2003. Available from: http://www.who.int/csr/sars/country/ country2003_08_15.pdf

5. World Health Organization (WHO). Consensus document on the epidemiology of severe acute respiratory syndrome (SARS) 2003. Geneva: WHO; 2003. Available from: http://www.who. int/csr/resources/publications/CDS_CSR_ARO_2004_2.pdf

6. Health Protection Agency (HPA). Infection control advice: Suspected or Confirmed Novel Coronavirus Cases. London:HPA; 2012. [Accessed 25 Sep 2012]. http://www.hpa. org.uk/webc/HPAwebFile/HPAweb_C/1317136232722

7. World Health Organization (WHO). WHO Case-finding interim case definition. Geneva: WHO; 2012. [Accessed 26 Sep 2012] Available from: http://www.who.int/csr/disease/coronavirus_ infections/en/index.html

8. World Health Organization (WHO). Case definition for case finding severe respiratory disease associated with novel coronavirus. Geneva: WHO; 2012. Available from: http://www. who.int/csr/disease/coronavirus_infections/en/index.html

9. Health Protection Agency (HPA). Partial genetic sequence information for scientists about the Novel Coronavirus 2012. London: HPA; 2012. [Accessed 25 Sep 2012]. Available from: http://www.hpa.org.uk/Topics/InfectiousDiseases/ InfectionsAZ/RespiratoryViruses/NovelCoronavirus/ respPartialgeneticsequenceofnovelcoronavirus 